\title{
High expression of SDF-1 and VEGF is associated with poor prognosis in patients with synovial sarcomas
}

\author{
QI FENG ${ }^{1}$, PENG GUO $^{1}$, JIN WANG ${ }^{1}$, XIAOYU ZHANG ${ }^{1}$, HUI-CHAI YANG ${ }^{2}$ and JIAN-GANG FENG ${ }^{1}$ \\ Departments of ${ }^{1}$ Orthopedics and ${ }^{2}$ Pathology, The Fourth Affiliated Hospital of \\ Hebei Medical University, Shijiazhuang, Hebei 050011, P.R. China
}

Received April 2, 2016; Accepted March 17, 2017

DOI: $10.3892 /$ etm.2018.5684

\begin{abstract}
Stromal cell-derived factor-1 (SDF-1) predicts poor clinical outcomes of certain types of cancer. Furthermore, vascular endothelial growth factor (VEGF) promotes the growth and metastasis of solid tumors. The aim of the present study was to examine the expression of SDF-1 and VEGF in patients with synovial sarcoma and to determine their expression is correlated with unfavorable outcomes. Levels of SDF-1 and VEGF proteins were evaluated in 54 patients with synovial sarcoma using immunohistochemical and immunofluorescence staining. Potential associations between the expression of SDF-1 and VEGF and various clinical parameters were analyzed using Pearson's $\chi^{2}$ test and the Spearman-rho test. Additionally, univariate and multivariate Cox regression analyses were used to identify potential prognostic factors, and the Kaplan-Meier method was used to analyze the overall survival rates of patients. Low SDF-1 and VEGF expression was detected in $20.4 \%$ (11/54) and $22.2 \%$ (12/54) of patients with synovial sarcoma; moderate expression was detected in $35.2 \%(19 / 54)$ and $37.0 \%(20 / 54)$ of patients and high expression was detected in $44.4 \%$ (24 of 54) and 40.7\% (22 of 54) of patients, respectively. Levels of SDF-1 and VEGF proteins were significantly associated with histological grade $(\mathrm{P}<0.05)$, metastasis $(\mathrm{P}<0.05)$ and American Joint Committee on Cancer staging $(\mathrm{P}<0.05)$. In addition, levels of SDF-1 and VEGF expression were positively correlated with each other $(\mathrm{P}<0.001)$. Univariate analysis also indicated that VEGF expression was associated with shorter overall survival rates in $(\mathrm{P}<0.05)$, whereas multivariate analysis demonstrated that SDF-1 expression was associated with shorter patient survival rates $(\mathrm{P}<0.05)$. Finally, both SDF-1 and VEGF expression were associated with various characteristics of synovial sarcoma.
\end{abstract}

Correspondence to: Dr Jian-Gang Feng, Department of Orthopedics, The Fourth Affiliated Hospital of Hebei Medical University, 12 Jiankang Road, Shijiazhuang, Hebei 050011, P.R. China

E-mail: fengjiangang0311@126.com

Key words: stromal cell-derived factor-1, vascular endothelial growth factor, synovial sarcoma, prognosis
Therefore, SDF-1 expression may be a potential independent prognostic indicator in patients with synovial sarcomas.

\section{Introduction}

Synovial sarcomas (SS) are clinically aggressive malignant tumors of mesenchymal origin and patients with SS are susceptible to early systemic metastases $(1,2)$. Although the long-term outcomes for patients undergoing surgery for SS have improved due to the development of systemic chemotherapy, the overall prognosis of patients with SS remains unsatisfactory $(3,4)$. SS are characterized by local recurrence and early lung metastases, and the 5-year survival rate for SS ranges between 20 and 30\% (5). Therefore, the establishment of efficient therapeutic strategies is required to improve the prognosis of such patients.

Chemotaxis is involved in many physiological processes, including stem-cell homing, hematopoiesis, extracellular matrix remodeling and cell-mediated wound healing (6-11). Previous studies have identified an association between tumorigenesis and chemokines (6-12). Thus, these chemokines such as CXCL may be useful as potential therapeutic targets to attenuate tumor progression.

Stromal cell-derived factor-1 (SDF-1), also known as CXCL-12, primarily regulates the progression of chemotaxis and may promote tumor formation (6-13). SDF-1 has been implicated in almost all malignant cancers, including breast, lung, and colon cancer, as well as tumors of hematopoietic origins (14). Furthermore, it has been demonstrated that SDF-1 increases the recurrence and metastasis of malignant tumors, as it may enhance the survival of tumor cells by preventing apoptosis $(15,16)$, resulting in decreased survival rates and unfavorable clinical outcomes in cancer patients.

Vascular endothelial growth factor (VEGF) is one of the most important cytokines in the human body and promotes neovascularization and carcinogenesis via SDF-1 signaling (17). Previous studies have demonstrated that overexpression of VEGF is essential for the growth and survival of many types of cancer cells (18). In addition, high levels of VEGF are associated with unfavorable survival rates in patients with SS $(18,19)$. The present study aimed to evaluate the expression patterns of SDF-1 and VEGF in samples from SS tissue and determine the potential association between SDF-1 and VEGF expression and patient clinical outcomes. 
Table I. Clinicopathological variables and the expression of SDF-1 and VEGF.

\begin{tabular}{|c|c|c|c|c|c|c|c|c|c|}
\hline \multirow[b]{2}{*}{ Characteristics } & & \multicolumn{4}{|c|}{ SDF-1 } & \multicolumn{4}{|c|}{ VEGF } \\
\hline & & $-/+(\%)$ & $++(\%)$ & $+++(\%)$ & P-value & $-/+(\%)$ & $++(\%)$ & $+++(\%)$ & P-value \\
\hline Sex & & & & & 0.202 & & & & 0.717 \\
\hline Female & 23 & $6(26.1)$ & $10(43.5)$ & $7(30.4)$ & & $6(26.1)$ & $9(39.1)$ & $8(34.8)$ & \\
\hline Male & 31 & $5(16.1)$ & $9(29.0)$ & $17(54.8)$ & & $6(19.4)$ & $11(35.5)$ & $14(45.2)$ & \\
\hline Age (years) & & & & & 0.217 & & & & 0.490 \\
\hline$\geq 30$ & 25 & $5(20.0)$ & $6(24.0)$ & $14(56.0)$ & & $4(16.0)$ & $11(44.0)$ & $10(40.0)$ & \\
\hline$<30$ & 29 & $6(20.7)$ & $13(44.8)$ & $10(34.5)$ & & $8(27.6)$ & $9(31.0)$ & $12(41.4)$ & \\
\hline Tumor size $(\mathrm{cm})$ & & & & & 0.609 & & & & 0.787 \\
\hline$<5$ & 32 & $6(18.8)$ & $10(31.3)$ & $16(50.0)$ & & $7(21.9)$ & $13(40.6)$ & $12(37.5)$ & \\
\hline$\geq 5$ & 22 & $5(22.7)$ & $9(40.9)$ & $8(36.4)$ & & $5(22.7)$ & $7(31.8)$ & $10(45.5)$ & \\
\hline Histological grade & & & & & 0.004 & & & & 0.042 \\
\hline I & 12 & $5(41.7)$ & $2(16.7)$ & $5(41.7)$ & & $6(50.0)$ & $4(33.3)$ & $2(16.7)$ & \\
\hline II & 20 & $2(10.0)$ & $13(65.0)$ & $5(25.0)$ & & $2(10.0)$ & $10(50.0)$ & $8(40.0)$ & \\
\hline III & 22 & $4(18.2)$ & $4(18.2)$ & 14 (63.6) & & $4(18.2)$ & $6(27.3)$ & $12(54.5)$ & \\
\hline Distant metastatis ${ }^{\mathrm{a}}$ & & & & & 0.009 & & & & 0.028 \\
\hline No & 34 & $11(32.4)$ & $12(35.3)$ & $11(32.4)$ & & $11(32.4)$ & $13(38.2)$ & $10(29.4)$ & \\
\hline Yes & 20 & $0(0.0)$ & $7(35.0)$ & $13(65.0)$ & & $1(5.0)$ & $7(35.0)$ & $12(60.0)$ & \\
\hline AJCC staging ${ }^{\mathrm{a}}$ & & & & & $<0.001$ & & & & 0.003 \\
\hline $\mathrm{I} / \mathrm{II}$ & 25 & $11(44.0)$ & $11(44.0)$ & $3(12.0)$ & & $10(40.0)$ & $10(40.0)$ & $5(20.0)$ & \\
\hline III/IV & 29 & $0(0.0)$ & 8 (27.6) & $21(72.4)$ & & $2(6.9)$ & $10(34.5)$ & $17(58.6)$ & \\
\hline
\end{tabular}

Pearson's $\chi^{2}$ test was used. ${ }^{\text {P }}<0.05$. SDF-1, stromal cell-derived factor-1; VEGF, vascular endothelial growth factor; AJCC, American Joint Committee on Cancer.

\section{Materials and methods}

Patients and samples. Paraffin-embedded specimens were collected from 54 patients who visited the Fourth Hospital of Hebei Medical University (Shijiazhuang, China) between January 2004 and December 2010. Clinical and histopathological characteristics, including sex, age, tumor size, histological grade, distant metastasis, AJCC staging, and information on patient follow-up and survival, were collected retrospectively. The specimens were fixed in $10 \%$ neutral-buffered formalin overnight at room temperature and then were achieved for later use. Furthermore, radiotherapy and chemotherapy were not administered prior to surgery in any patient. All of the data were grouped according to the patient's age, sex, tumor size ( $<5$ vs. $\geq 5 \mathrm{~cm}$ ) and histological cancer profile. Each patient was assigned a histological grade according to the Fédération Nationale des Centres de Lutte Contre le Cancer (20), the presence of distant metastases and American Joint Committee on Cancer (AJCC) staging (21). The present study was approved by Ethics Committee of The Fourth Affiliated Hospital of Hebei Medical University (Shijiazhuang, China) and informed written consent was obtained from all patients.

Immunohistochemical and immunofluorescence staining and scoring. For immunohistochemical analysis, a tissue microarray was produced using 4.0-mm diameter tumor cores with 1 core per case. Antigen retrieval was performed by microwaving the array in sodium citrate buffer at $95^{\circ} \mathrm{C}$ for $10 \mathrm{~min}$. Subsequently, the samples were blocked in normal goat serum (Sigma-Aldrich; Merck KGaA, Darmstadt, Germany) at $37^{\circ} \mathrm{C}$ for $1 \mathrm{~h}$ Immunohistochemical staining was performed according to a routine protocol (18). Samples were incubated at $37^{\circ} \mathrm{C}$ for $1 \mathrm{~h}$ with rabbit anti-human SDF-1 (cat. no. GTX116092, 1:100; Bethyl Laboratories, Montgomery, Inc., TX, USA) and rabbit anti-human VEGF (cat. no. sc-152, 1:50; Santa Cruz Biotechnology, Inc., Dallas, TX, USA). Following this, samples were incubated with horseradish peroxidase-conjugated secondary antibody (cat. no. A6667, Sigma-Aldrich; Merck KGaA) at $37^{\circ} \mathrm{C}$ for $1 \mathrm{~h}$. Stained sections were analyzed under an optical microscope by three pathologists who were blinded to the patient data. The mean number of immunopositive cells in the samples was determined in 5 random fields-of-view at a magnification of $\mathrm{x} 400$. Furthermore, immunohistochemical results were evaluated according to the Friedrich' immunoreactivity score (IRS) (18) based on two categories: The percentage of stained cells $(\mathrm{X}):<1 \%$ (score $=0) ; 1-25 \%($ score $=1) ; 25-50 \%$ (score $=2)$; $51-80 \%$ (score $=3) ;>80 \%$ (score $=4)$; the staining intensity $(\mathrm{Y})$ : no staining (score $=0)$; buff (score $=1)$; darker buff (score $=2$ ); $\tan$ (score=3). X x Y was calculated as the final score, and staining was described as low (final score $0-3,-/+$ ), moderate (final score $4-7,++$ ) or high (final score $>7,+++$ ). Analysis was performed using ImagePro Plus software (version 6; Media Cybernetics, Rockville, MD, USA). 
Table II. Correlation between SDF-1 and VEGF expression.

\begin{tabular}{|c|c|c|c|c|c|}
\hline \multirow[b]{2}{*}{ Characteristics } & & \multicolumn{3}{|c|}{ SDF-1 } & \multirow[b]{2}{*}{ P-value (Spearman) } \\
\hline & & $-/+(\%)$ & $++(\%)$ & $+++(\%)$ & \\
\hline $\mathrm{VEGF}^{\mathrm{a}}$ & & & & & $<0.001$ \\
\hline$-/+$ & 12 & $8(66.7)$ & $3(25.0)$ & $1(8.3)$ & \\
\hline++ & 20 & $2(10.0)$ & $11(55.0)$ & $7(35.0)$ & \\
\hline+++ & 22 & $1(4.5)$ & $5(22.7)$ & $16(72.7)$ & \\
\hline Total & 54 & 11 & 19 & 24 & \\
\hline
\end{tabular}

Spearman-rho test was used. ${ }^{a} \mathrm{P}<0.05$. Stromal cell-derived factor-1; VEGF, vascular endothelial growth factor.

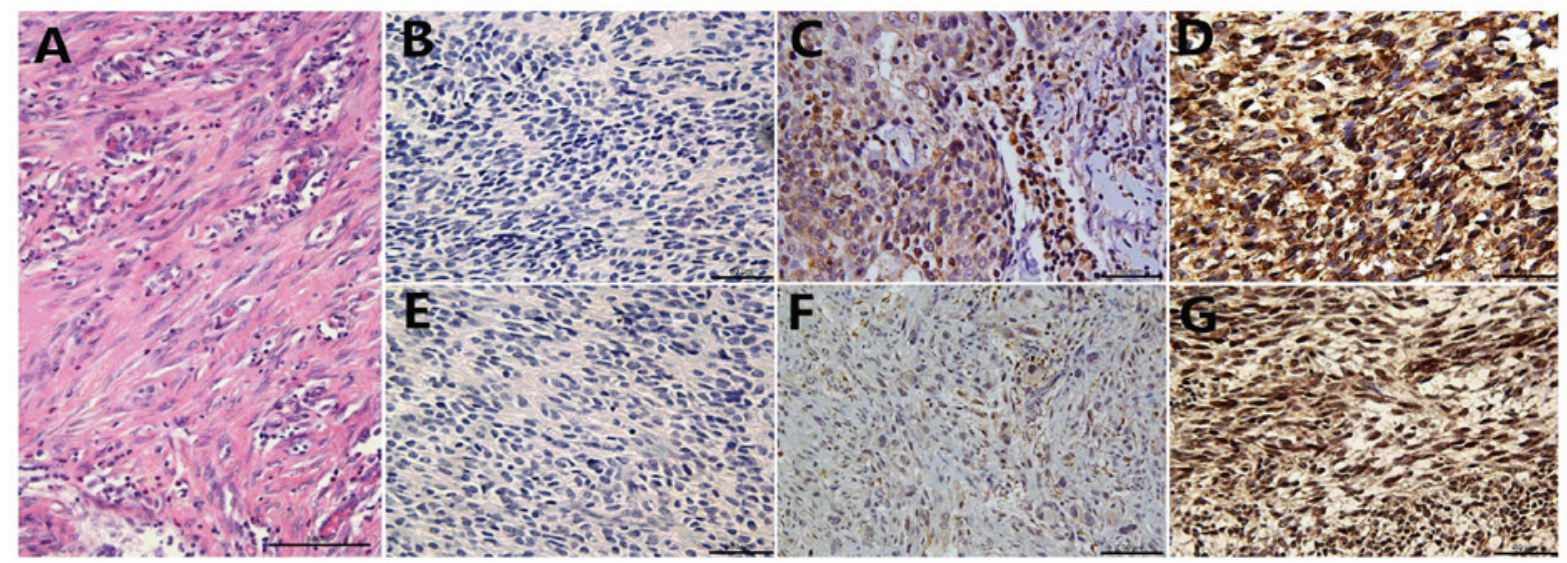

Figure 1. Immunohistochemical staining of SDF-1 and VEGF in SS specimens. (A) Hematoxylin and eosin staining of SS specimen. Immunohistochemical staining of SDF-1 was: (B) low, (C) moderate, and (D) high. Immunohistochemical staining of VEGF was: (E) low, (F) moderate, and (G) high. Magnification, x400. SS, synovial sarcomas; SDF-1, stromal cell-derived factor-1; VEGF, vascular endothelial growth factor. Scale bar, $50 \mu \mathrm{m}$.

Statistical analyses. The end of the follow-up period was defined as either the date of patient mortality or the patient's last date of contact, up to January 2015. Overall survival (OS) was defined as the period of time from the date of the diagnosis to the date of last contact or patient mortality. Furthermore, the association of potential prognostic factors with SEF-1 and VEGF expression was analyzed using the $\chi^{2}$ test. For the correlation analysis, the Spearman-rho test was used to compare histological and clinical variables. Univariate and multivariate analysis for the potential prognostic factors and the OS was conducted using the Cox proportional hazards regression analysis. Additionally, the Kaplan-Meier curve method was used to determine the OS. SPSS software (version 22.0; IBM SPSS, Armonk, NY, USA) was used for statistical analysis and $\mathrm{P}<0.05$ was considered to indicate a statistically significant difference.

\section{Results}

Patient characteristics. Patient characteristics are presented in Table I. The mean patient age was $56.2 \pm 18.4$ years (range, 25-74 years) and the median OS was 11 months (range, 3-83 months).

Association between SDF-1 and VEGF expression levels and clinicopathological characteristics. Associations between
SDF-1 and VEGF and clinicopathological characteristics are summarized in Table I. Typical SDF-1 and VEGF staining in SS tissues are presented in Fig. 1. It was determined that in SS tissues, SDF-1 expression was low in $20.4 \%$ (11/54), moderate in $35.2 \%$ (19/54) and high in $44.4 \%(24 / 54)$ of cases, whereas VEGF expression was low in $22.2 \%$ (12/54), moderate in $37.0 \%$ (20/54) and high in 40.7\% (22/54) of cases. Additionally, both SDF-1 and VEGF expression were significantly associated with histological grade $(\mathrm{P}<0.05)$, distant metastasis $(\mathrm{P}<0.05)$ and AJCC staging $(\mathrm{P}<0.05)$. No significant associations were identified between SDF-1 and VEGF expression levels and other clinicopathological features (Table I).

SDF-1 expression is positively correlated with VEGF expression. The expression of SDF-1 was significantly correlated with VEGF expression $(\mathrm{P}<0.001)$, with a correlation coefficient of 0.618 (Table II). Furthermore, immunofluorescence analysis of paraffin-embedded specimens determined that SDF-1 and VEGF were located at the appropriate sections in SS cells (Fig. 2).

High expression of SDF-1 and VEGF in patients with SS correlates with poor $O S$. Univariate Cox proportional hazard analyzes for OS are summarized in Table III and Kaplan-Meier curves are presented in Fig. 3. Sex, age, tumor 


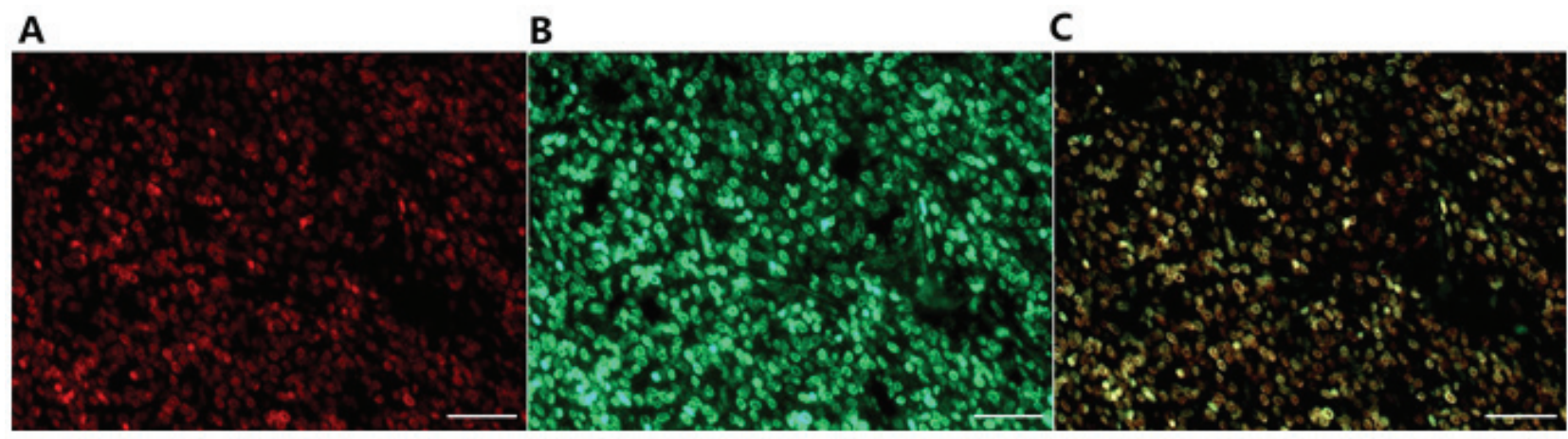

Figure 2. Double immunofluorescence staining of SDF-1 and VEGF in SS specimens. (A) Immunofluorescence staining of SDF-1. (B) Immunofluorescence staining of VEGF. (C) Merged immunofluorescence staining of SDF-1 and VEGF. Magnification, x400. SS, synovial sarcomas; SDF-1, stromal cell-derived factor-1; VEGF, vascular endothelial growth factor. Scale bar, $50 \mu \mathrm{m}$.

A

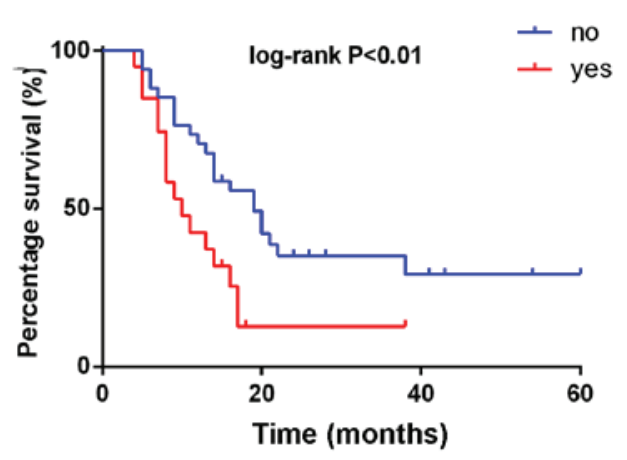

C

Expression of VEGF

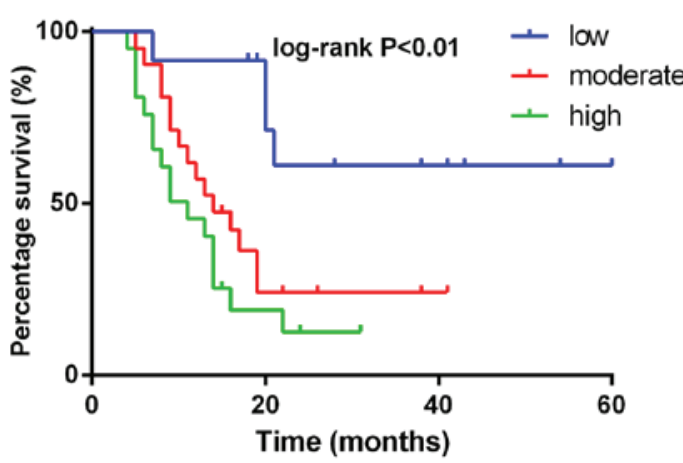

\section{B Expression of SDF-1}

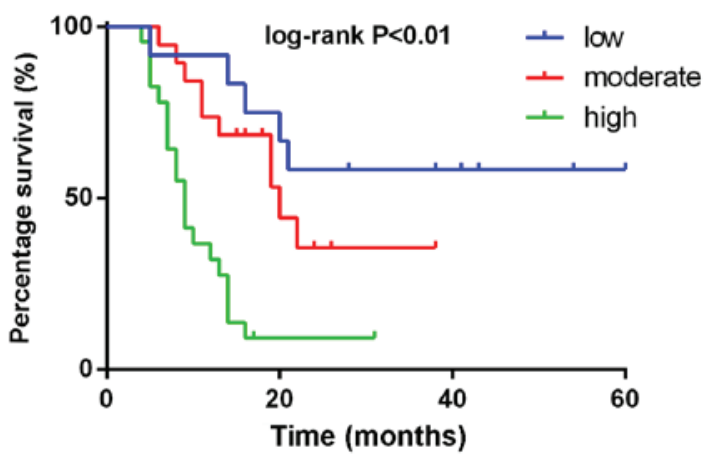

D

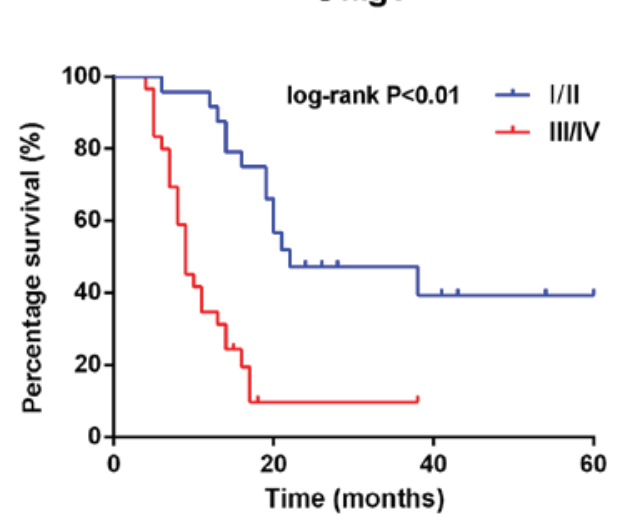

Figure 3. Overall survival curves of patients with SS. (A) Association of OS with distant metastasis, (B) association of OS with SDF-1 expression, (C) association of OS with VEGF expression and (D) association of OS with AJCC staging. P-values were determined by comparing survival distributions using the log-rank test. SS, synovial sarcomas; SDF-1, stromal cell-derived factor-1; VEGF, vascular endothelial growth factor; OS, overall survival; AJCC, American Joint Committee on Cancer.

size and histological grade were not significant in predicting OS. However, distant metastasis $(\mathrm{P}<0.01)$ and higher AJCC staging $(\mathrm{P}<0.01)$ predicted shorter OS (Fig. 3). In addition, univariate analysis revealed that SDF-1 $(\mathrm{P}<0.05)$ and VEGF $(\mathrm{P}<0.01)$ expression were significantly associated with shorter OS (Table III; Fig. 3).

SDF-1 expression is an independent prognostic factor for poor overall survival of SS patients. Univariate factors associated with OS were identified by a multivariate Cox proportional hazard (Table IV). Distant metastasis $[\mathrm{P}=0.017$, $\mathrm{HR}=0.185$ (0.046-0.737)], AJCC staging $[\mathrm{P}=0.04, \mathrm{HR}=3.680$ (1.519-8.914)] and SDF-1 expression $[\mathrm{P}=0.026, \mathrm{HR}=2.640$ (1.124-6.200)] were independent prognostic factors for OS. Furthermore, SS patients with higher SDF-1 expression exhibited a significantly greater risk of mortality $\left(\mathrm{P}_{\text {log-rank }}<0.01\right)$ than patients with lower SDF-1 expression (Fig. 3). Therefore, the expression of SDF-1 appears to be a 
Table III. Univariate Cox proportional regression analysis of clinicopathological factors associated with OS.

\begin{tabular}{|c|c|c|c|c|}
\hline \multirow[b]{2}{*}{ Characteristics } & & \multicolumn{3}{|c|}{ OS } \\
\hline & & HR & $95 \% \mathrm{CI}$ & P-value \\
\hline \multicolumn{5}{|l|}{ Sex } \\
\hline Female & 23 & 1 & - & - \\
\hline Male & 31 & 1.543 & $0.810-2.936$ & 0.187 \\
\hline \multicolumn{5}{|l|}{ Age (years) } \\
\hline$\geq 30$ & 25 & 1 & - & - \\
\hline$<30$ & 29 & 1.062 & $0.569-1.982$ & 0.851 \\
\hline \multicolumn{5}{|l|}{ Tumor size $(\mathrm{cm})$} \\
\hline$<5$ & 32 & 1 & - & - \\
\hline$\geq 5$ & 22 & 0.924 & $0.492-1.736$ & 0.806 \\
\hline \multicolumn{5}{|l|}{ Histological grade } \\
\hline $\mathrm{I}$ & 12 & 1 & - & 0.251 \\
\hline II & 20 & 1.105 & $0.471-2.589$ & 0.819 \\
\hline III & 22 & 1.802 & $0.802-4.048$ & 0.154 \\
\hline \multicolumn{5}{|c|}{ Distant metastasis ${ }^{\mathrm{a}}$} \\
\hline No & 56 & 1 & - & - \\
\hline Yes & 33 & 2.452 & $1.247-4.820$ & 0.009 \\
\hline \multicolumn{5}{|l|}{ AJCC staging ${ }^{\mathrm{a}}$} \\
\hline I & 9 & 1 & - & $<0.001$ \\
\hline II & 16 & 3.517 & $1.013-12.210$ & 0.048 \\
\hline III & 11 & 22.10 & $5.371-90.908$ & $<0.001$ \\
\hline IV & 18 & 33.33 & $6.599-139.440$ & $<0.001$ \\
\hline \multicolumn{5}{|l|}{ SDF-1 ${ }^{\mathrm{a}}$} \\
\hline Low $(-/+)$ & 11 & 1 & - & $<0.001$ \\
\hline Moderate (++) & 19 & 3.165 & $1.042-9.610$ & 0.042 \\
\hline High $(+++)$ & 24 & 18.73 & $5.496-63.880$ & $<0.001$ \\
\hline \multicolumn{5}{|l|}{ VEGF $^{\mathrm{a}}$} \\
\hline Low $(-/+)$ & 12 & 1 & - & $<0.001$ \\
\hline Moderate (++) & 20 & 3.305 & $1.265-8.632$ & 0.015 \\
\hline High $(+++)$ & 22 & 4.826 & $1.825-12.760$ & 0.002 \\
\hline
\end{tabular}

${ }^{\mathrm{a}} \mathrm{P}<0.05$. OS, overall survival; HR, hazard ratio; CI, confidence interval; SDF-1, stromal cell-derived factor-1; VEGF, vascular endothelial growth factor; AJCC, American Joint Committee on Cancer; OS, overall survival.

Table IV. Multivariate Cox regression analysis of clinicopathological factors associated with OS.

\begin{tabular}{lccc}
\hline & \multicolumn{3}{c}{ OS } \\
\cline { 2 - 4 } Factors & HR & $95 \%$ CI & P-value \\
\hline AJCC staging $^{\mathrm{a}}$ & 3.680 & $1.519-8.914$ & 0.004 \\
Distant metastasis $^{\mathrm{a}}$ & 0.185 & $0.046-0.737$ & 0.017 \\
SDF-1 $^{\mathrm{a}}$ & 2.640 & $1.124-6.200$ & 0.026 \\
\hline
\end{tabular}

${ }^{\mathrm{a}} \mathrm{P}<0.05$. OS, overall survival; $\mathrm{HR}$, hazard ratio; $\mathrm{CI}$, confidence interval; AJCC, American Joint Committee on Cancer; SDF-1, stromal cell-derived factor-1. potentially significant clinical prognostic factor in patients with SS.

\section{Discussion}

To the best of our knowledge, this is the first study to demonstrate that levels of SDF-1 and VEGF expression are correlated with the occurrence of SS. In addition, it was determined that high SDF-1 and VEGF expression was significantly associated with unfavorable clinical variables. Furthermore, SDF-1 expression was positively correlated with VEGF expression, and SDF-1 expression alone was sufficient to be an independent prognostic indicator of OS in multivariate Cox regression analysis. Overall, the results of the present study demonstrate 
that both SDF-1 and VEGF expression may be significant prognostic factors for SS and result in unfavorable clinical outcomes in patients.

SDF-1 is one of the CXCs family chemokines and is important in chemotaxis, stem cell homing, self-renewal and differentiation, hematopoiesis and wound healing $(3,12,22-24)$. Tumor cells are capable of overexpressing chemokine receptors and chemokines may be important in cancer progression and organ-selective metastasis $(14,25)$. It has been hypothesized that disseminated tumor cells expressing chemokine receptors can invade the circulation and are subsequently attracted and arrested by their corresponding ligands. The local/original and specific metastatic sites initiate an inflammatory response in nearby tissues, resulting in the expression of additional chemokines (26). These chemokines are then able to induce the procession, recurrence, and migration of tumor cells. A number of studies have demonstrated that SDF-1 is important in various types of cancers, including prostate, ovarian and breast tumors $(12,13,27)$. The observations of the present study are consistent with those from previous studies and confirm that SDF-1 expression is associated with poor clinical outcomes.

In original and/or metastatic tumor sites, reconstruction of local microvascular environment is one of the most important steps facilitating the survival of new mitotic tumor cells (28). It is thought that SDF-1 may upregulate the expression of VEGF via the SDF-1/CXCR-4 (chemokine receptor-4, the specific receptor of SDF-1) pathway (29). Furthermore, overexpression of VEGF in tumor cells may lead to aggressive tumorigenesis and distant metastasis, resulting in poor clinical outcomes $(30,31)$. The present study revealed that there is a significant association between the levels of SDF-1 and VEGF expression in SS. A strong association between levels of SDF-1 and VEGF expression and lower histological grade, higher stage, increased distant metastasis and poor prognosis in patients with SS was also identified. To the best of our knowledge, these results provide the first evidence that SDF-1 and VEGF are involved in SS, which is consistent with previous studies (32). Therefore, SDF-1 is not only a potential prognostic marker, but also a novel target for therapeutic intervention in patients with SS. However, the exact role of SDF-1 and VEGF in SS has not been fully elucidated, and additional in vivo and in vitro investigations of the molecular mechanisms are required.

There were a number of limitations in the present study. For example, immunohistochemistry and immunofluorescence were semi-quantitative and not as accurate as reverse transcription-quantitative polymerase chain reaction or western blot analysis would have been. Therefore, some bias may have been introduced. However, the samples were analyzed in a blinded fashion by three pathologists, and a consensus was reached by discussion if disagreements occurred. The sample size of the present study was also relatively small, meaning that the results would need to be confirmed in a larger population.

In conclusion, a significant proportion of patients with SS exhibited high expression of SDF-1 and VEGF. Expression of SDF-1 and VEGF was associated with unfavorable clinical characteristics and poor prognosis of SS patients. Although the role of SDF-1 and VEGF in SS remains unclear, SDF-1 appears to be a significant potential clinical prognostic factor in patients with SS.

\section{References}

1. Nielsen TO, Poulin NM and Ladanyi M: Synovial sarcoma: Recent discoveries as a roadmap to new avenues for therapy. Cancer Discov 5: 124-134, 2015.

2. Spillane AJ, A'Hern R, Judson IR, Fisher C and Thomas JM: Synovial sarcoma: A clinicopathologic, staging, and prognostic assessment. J Clin Oncol 18: 3794-3803, 2000.

3. Wunder JS, Nielsen TO, Maki RG, O'Sullivan B and Alman BA: Opportunities for improving the therapeutic ratio for patients with sarcoma. Lancet Oncol 8: 513-524, 2007.

4. Thway K and Fisher C: Synovial sarcoma: Defining features and diagnostic evolution. Ann Diagn Pathol 18: 369-380, 2014.

5. Palmerini E, Paioli A and Ferrari S: Emerging therapeutic targets for synovial sarcoma. Expert Rev Anticancer Ther 14: 791-806, 2014.

6. Murphy PM: Chemokines and the molecular basis of cancer metastasis. N Engl J Med 345: 833-835, 2001.

7. Kuil J, Buckle T and van Leeuwen FW: Imaging agents for the chemokine receptor 4 (CXCR4). Chem Soc Rev 41: 5239-5261, 2012.

8. Ruiz de Almodovar C, Luttun A and Carmeliet P: An SDF-1 trap for myeloid cells stimulates angiogenesis. Cell 124: 18-21, 2006.

9. Kiel MJ and Morrison SJ: Maintaining hematopoietic stem cells in the vascular niche. Immunity 25: 862-864, 2006.

10. Ratajczak MZ, Kim CH, Abdel-Latif A, Schneider G, Kucia M, Morris AJ, Laughlin MJ and Ratajczak J: A novel perspective on stem cell homing and mobilization: Review on bioactive lipids as potent chemoattractants and cationic peptides as underappreciated modulators of responsiveness to SDF-1 gradients. Leukemia 26: 63-72, 2012.

11. Klein RS and Rubin JB: Immune and nervous system CXCL12 and CXCR4: Parallel roles in patterning and plasticity. Trends Immunol 25: 306-314, 2004.

12. Epstein RJ: The CXCL12-CXCR4 chemotactic pathway as a target of adjuvant breast cancer therapies. Nat Rev Cancer 4: 901-909, 2004

13. Burger JA and Kipps TJ: CXCR4: A key receptor in the crosstalk between tumor cells and their microenvironment. Blood 107: 1761-1767, 2006.

14. Sun X, Cheng G, Hao M, Zheng J, Zhou X, Zhang J, Taichman RS, Pienta KJ and Wang J: CXCL12/CXCR4/CXCR7 chemokine axis and cancer progression. Cancer Metastasis Rev 29: 709-722, 2010.

15. Yao C, Li P, Song H, Song F, Qu Y, Ma X, Shi R and Wu J: CXCL12/CXCR4 axis upregulates twist to induce EMT in human glioblastoma. Mol Neurobiol 53: 3948-3953, 2016.

16. Yang P, Wang G, Huo H, Li Q, Zhao Y and Liu Y: SDF-1/CXCR4 signaling up-regulates survivin to regulate human sacral chondrosarcoma cell cycle and epithelial-mesenchymal transition via ERK and PI3K/AKT pathway. Med Oncol 32: 377, 2015.

17. Chen Y, Gou X, Kong DK, Wang X, Wang J, Chen Z, Huang C and Zhou J: EMMPRIN regulates tumor growth and metastasis by recruiting bone marrow-derived cells through paracrine signaling of SDF-1 and VEGF. Oncotarget 6: 32575-32585, 2015.

18. Hassan S, Buchanan M, Jahan K, Aguilar-Mahecha A, Gaboury L, Muller WJ, Alsawafi Y, Mourskaia AA, Siegel PM, Salvucci O and Basik M: CXCR4 peptide antagonist inhibits primary breast tumor growth, metastasis and enhances the efficacy of anti-VEGF treatment or docetaxel in a transgenic mouse model. Int J Cancer 129: 225-232, 2011.

19. Eswarappa SM and Fox PL: Antiangiogenic VEGF-Ax: A new participant in tumor angiogenesis. Cancer Res 75: 2765-2769, 2015.

20. Sápi Z: Pathology of soft tissue sarcomas. Magy Onkol 58: 11-23, 2014 (In Hungarian)

21. Kneisl JS, Coleman MM and Raut CP: Outcomes in the management of adult soft tissue sarcomas. J Surg Oncol 110: 527-538, 2014.

22. Sánchez-Martín L, Sánchez-Mateos P and Cabañas C: CXCR7 impact on CXCL12 biology and disease. Trends Mol Med 19: 12-22, 2013.

23. Woodard LE and Nimmagadda S: CXCR4-based imaging agents. J Nucl Med 52: 1665-1669, 2011.

24. Barbieri F, Thellung S, Würth R, Gatto F, Corsaro A, Villa V, Nizzari M, Albertelli M, Ferone D and Florio T: Emerging targets in pituitary adenomas: Role of the CXCL12/CXCR4-R7 system. Int J Endocrinol 2014: 753524, 2014.

25. Chatterjee S, Behnam Azad B and Nimmagadda S: The intricate role of CXCR4 in cancer. Adv Cancer Res 124: 31-82, 2014. 
26. Mao AW, Jiang TH, Sun XJ and Peng J: Application of chemokine receptor antagonist with stents reduces local inflammation and suppresses cancer growth. Tumour Biol 36: 8637-8643, 2015

27. Lee HJ and Jo DY: The role of the CXCR4/CXCL12 axis and its clinical implications in gastric cancer. Histol Histopathol 27: 1155-1161, 2012.

28. García-Román J and Zentella-Dehesa A: Vascular permeability changes involved in tumor metastasis. Cancer Lett 335: 259-269, 2013.

29. Teicher BA and Fricker SP: CXCL12 (SDF-1)/CXCR4 pathway in cancer. Clin Cancer Res 16: 2927-2931, 2010.
30. Kampmann E, Altendorf-Hofmann A, Gibis S, Lindner LH, Issels R, Kirchner T and Knösel T: VEGFR2 predicts decreased patients survival in soft tissue sarcomas. Pathol Res Pract 211: 726-730, 2015.

31. Ben-Baruch A: The multifaceted roles of chemokines in malignancy. Cancer Metastasis Rev 25: 357-371, 2006.

32. Kawauchi S, Fukuda T and Tsuneyoshi M: Angiogenesis does not correlate with prognosis or expression of vascular endothelial growth factor in synovial sarcomas. Oncol Rep 6: 959-964, 1999. 